\title{
Biometría dactilar: una nueva alternativa de controlar efectivamente la asistencia a clases
}

\section{Fingerprint biometry: a new way to effectively control class attendance}

Jhon Alexander Calderón Velasco ${ }^{1}$, Gloria Alexandra Amarillo Cárdenas², Luis Alejandro Silva Bahamon³, Carlos Gilberto Donoso Albarracín ${ }^{4}$

\section{Resumen}

Objetivo: implementación de una alternativa de control de asistencia a clases, mediante biometría dactilar en la Fundación Universitaria UNINPAHU.

Metodología: mediante aplicación de encuestas y percepción a los estudiantes se obtuvieron datos relevantes y de acuerdo con estos se efectúo un análisis y diagnóstico que mostró la necesidad de utilizar tecnología para evolucionar de los formatos en papel al registro de la asistencia por medio de la huella dactilar.

Resultados: se identificó, diseñó y desarrolló una aplicación de software para llevar el control por medio de biometría, lo que evidenció una optimización del proceso, disminución del uso de papel, centralización de los datos y la disponibilidad de la información en línea, para apoyar la toma de decisiones administrativas y académicas.

Conclusiones: la investigación determinó la percepción de los estudiantes de la Universidad sobre el proceso de asistencia a clase y la importancia de cambiar a otras metodologías, que reduzcan los costos del uso de papelería, percepciones que se consideraron en la presente investigación y condujeron al producto final.

\section{Abtract}

Objective: implementation of an alternative of attendance control to classes, by means of fingerprint biometrics in the University Foundation UNINPAHU.

Methodology: through the application of surveys and perception to the students, relevant data were obtained and, according to these, an analysis and diagnosis was made that showed the need to use technology to evolve from the paper formats to the record of attendance through the footprint. fingerprint

Results: a software application was identified, designed and developed to take control by means of biometrics, which evidenced an optimization of the process, a decrease in the use of paper, centralization of data and the availability of information online, to support making administrative and academic decisions.

Conclusions: the investigation determined the perception of the students of the University on the process of attendance to class and the importance of changing to other methodologies, that reduce the costs of the use of stationery, perceptions that were considered in the present investigation and led to the product final.

1 Fundación Universitaria Uninpahu, Correo electronico: jcalderonve01@uninpahu.edu.co

2 Fundación Universitaria Uninpahu, Correo electronico: gamarilloca@uninpahu.edu.co

3 Fundación Universitaria Uninpahu, Correo electronico: Isilvaba@uninpahu.edu.co

4 Autor de Correspondencia,Fundación Universitaria Uninpahu, Correo electronico: cdonosoal@uninpahu.edu.co

\section{Palabras} Claves:

Biometría, control, huella dactilar, reconocimiento, seguridad, software.

\section{Keywords:}

Biometrics, control, fingerprint, recognition, security, software. 


\section{Introducción}

Este articulo está basado en una investigación exploratoria realizada al sistema de control de asistencia a clase que llevaba la Fundación Universitaria UNINPAHU. Así, tras un análisis de los resultados, el estudio de diferentes alternativas de control de asistencia y la ejecución de un proyecto, se propuso una solución alternativa para el mejoramiento del proceso actual, mediante la elaboración de una plataforma que funciona a través de sistemas biométricos dactilares.

\section{Naturaleza de la investigación}

Es importante mencionar y contextualizar el proceso que actualmente desarrolla la Universidad UNINPAHU en lo pertinente al control de asistencia a clases. Este proceso comprende el uso de papelería impresa, la cual es distribuida a los docentes para ser diligenciada en cada una de las clases por los estudiantes. La información debe ser recopilada y entregada al área administrativa.

La problemática fundamental al respecto la constituye primero el gasto en papelería, así como falencias que se presentan en casos como el no registro de firmas a tiempo, olvido por parte de los profesores de los listados en el momento de la clase, la falsificación de firmas y la falta de reportes oportunos de la información.

Debido a lo anterior, se procedió a buscar alternativas para mitigar esta problemática y fue cuando se tomaron en cuenta los sistemas biométricos como alternativa tecnológica.

Como base de la investigación, también se consideraron investigaciones realizadas previamente en diferentes instituciones educativas como la Universidad de Guayaquil, con la implementación de un sistema biométrico para el control de empleados [1], la Universidad de La Salle, concretamente con un estudio de factibilidad para el control de acceso biométrico, en una empresa de empleados con huella dactilar [2] y, por último, un sistema biométrico implementado para hogares [3].

\section{Marco teórico}

El libro Biometrías 2 de la presidencia de la república de Argentina y desarrollado por la oficina tecnológica de esa nación en 2011 consolidó información del VI Congreso Internacional de Biometría en Argentina y recopiló artículos realizados por 15 investigadores que muestran diferentes puntos de vista y aplicaciones de la biometría [4].

Los sistemas biométricos se utilizan en gran medida a partir de dos pilares, identificación y autenticación. El primero se basa en un sistema uno a muchos, con el que se pretende identificar una persona y compararla con una base de datos; por su parte, la autenticación comprende un sistema de uno a uno y es en sí la comparación de un dato con otro dato guardado previamente, fiel copia del mismo. Por otra parte, las premisas fundamentales en la funcionalidad de la Biometría son tres: 
¿la persona es la que dice ser?, ¿la persona no es quien dice ser?, ¿puede ser la persona identificada con los datos en sistema? [5].

La Biometría es catalogada como una ciencia y su campo de estudio no es nuevo en el mundo. Los términos de Biometría vienen del latín bios, Vida y metria, medidas. Por tanto, los datos biométricos son construidos con base en medidas y características físicas y morfológicas manuales o automatizadas.

Para entender mejor el objeto del estudio realizado, se debe hacer referencia al origen histórico del concepto de biometría. Este se le atribuye a Pearson y Weldon y el primer antecedente histórico registrado data de 1858. En su libro The origin of finger-printing, Herschel estampó la huella de la palma de la mano de todos y cada uno de sus trabajadores al reverso de los contratos, a fin de identificarlos respecto a otras personas que podrían exigir el pago de sus actividades [6].

En esos años los temas relacionados con la evolución del ser humano tenían gran trascendencia, por lo que la investigación sobre la identificación de personas, enfocada en la biometría forense, avanzó notoriamente en la intención de identificar seres humanos a partir de la medición del cráneo y demás partes del cuerpo.

En 1891, el croata Vucetich perfeccionó el sistema galtoniano, que se basaba en un sistema de clasificación básico de huellas dactilares, y en 1896 se identificaría la clasificación lógica de diez registros dactilares basados en los patrones de las huellas dactilares.

Ahora bien, la justificación respecto al establecimiento y uso de un sistema de identificación para el control de asistencia a clases radica en las bondades del sistema biométrico, que se concretan en las respuestas a los siguientes interrogantes retomados del libro Biometría 2: 1. ¿Por qué tener un sistema biométrico? Para descubrir la identidad de una persona o verificar dicha identidad, dado que los sistemas biométricos están diseñados para esta función; 2 . ¿Es la persona la que dice ser? Bajo las metodologías de búsqueda de uno a uno y uno a muchos, es posible responder esta inquietud. De este modo, al hablar de uno a uno se hace referencia a la posibilidad de verificar la identificación cuando se realiza la verificación de un registro capturado y compararlo con un registro guardado previamente; en tanto que la metodología uno a muchos realiza la misma operación con la diferencia de que en este caso la comparación se lleva a cabo con muchos registros almacenados en una base de datos previa; 3. ¿La persona no es quien dice ser? En este caso, al realizar una comparación entre el registro y los datos almacenados, y no encontrar archivos coincidentes se establece un registro negativo; 4. ¿Es confiable la información? Sí es confiable, dado que cada persona nace con características únicas y medibles como las huellas dactilares, el iris, la configuración del rostro y otras características particulares.

Tomando como base la biometría dactilar expuesta y la investigación exploratoria se realizaron encuestas, en las que se evidenció la percepción de los estudiantes y docentes en el proceso de control que se lleva en la Universidad por medio de formatos impresos. Luego se analizaron los resultados mediante un diagnóstico que evidenció la necesidad de utilizar tecnología de biometría dactilar.

Como citar este artículo: J.A. Calderón, G. Amarillo, L. Silva., y C. Donoso, "Biometría dactilar: una nueva alternativa de controlar efectivamente la asistencia a clases", Investigación e Innovación en Ingenierias, vol. 6, n. 1, pp. 27 - 39., 2018. DOl:https://10.17081/invinno.6.1.2773 


\section{Objetivos}

Las anteriores respuestas soportaron el propósito principal de este trabajo, que consiste en identificar, diseñar y desarrollar una aplicación para llevar el control de asistencia de la Universidad UNINPAHU por medio de biometría, además de utilizar metodologías ágiles para el proceso de construcción del mismo.

Los objetivos específicos del presente trabajo, derivado de la investigación realizada, fueron: Diagnosticar las necesidades en el proceso de registro y control de asistencia a clase; identificar las opciones que cumplan o se adecuen a los resultados del diagnóstico realizado; exponer la metodología utilizada para la ejecución del proyecto realizado en la Fundación Universitaria UNINPAHU, explicar la funcionalidad del sistema biométrico y sus bondades en la Fundación Universitaria UNINPAHU.

\section{Metodología}

Para encontrar el sistema biométrico más adecuado se planteó una relación de semejanza entre las principales tecnologías biométricas que se expone en los siguientes cuadros comparativos. Estas fueron retomadas y complementadas del proyecto investigativo Sistemas Biométricos de Huella Dactilar. También se consideró la investigación “La experiencia colombiana en identificación biométrica aplicada a las elecciones", realizada por la Registraduría Nacional del Estado Civil, en la cual se afirma que la biometría es una tecnología de seguridad que mide características morfológicas como forma del rostro, iris, la voz o huella dactilar [7].

Tabla 1.

Ventajas y desventajas de reconocimiento

facial

Fuente: [7] elaboración propia

\begin{tabular}{|l|l|}
\hline \multicolumn{2}{|c|}{ Reconocimiento Facial } \\
\hline \multicolumn{1}{|c|}{ Ventajas } & \multicolumn{1}{c|}{ Desventajas } \\
\hline $\begin{array}{l}\text { Sistema de reconocimiento no invasivo por local } \\
\text { que no requiere contacto con el autentificador }\end{array}$ & $\begin{array}{l}\text { Vareación en el ángulo de la cabeza lo que } \\
\text { no permite realizar el proceso de verificación }\end{array}$ \\
\hline $\begin{array}{l}\text { Las personas pueden estar en movimiento cuando } \\
\text { seencuentran frenteal sistema biométrico }\end{array}$ & $\begin{array}{l}\text { Cambios de iluminación pueden llegar a } \\
\text { afectar el sistema biométrico }\end{array}$ \\
\hline $\begin{array}{l}\text { Alta aceptabilidad ya que los usuarios no ven } \\
\text { interrumpidos su flujo de acceso y trabajo }\end{array}$ & $\begin{array}{l}\text { Los gestos expresivos pueden alterar el } \\
\text { proceso de identificación. }\end{array}$ \\
\hline $\begin{array}{l}\text { Tipo de sistema biométrico es utilizado } \\
\text { para entre humano entre varios individuos }\end{array}$ & $\begin{array}{l}\text { Obstrucción debido las lentes, gafas o } \\
\text { sombreros que puedan cubrir parte de la cara }\end{array}$ \\
\hline \multicolumn{1}{|c|}{$\begin{array}{l}\text { Es un sistema no invasivo ya que } \\
\text { únicamente se trata de una fotografía }\end{array}$} & $\begin{array}{l}\text { Uno de los mayores problemas es la utili- } \\
\text { zación de máscaras, por lo que es dificil de } \\
\text { ser detectado por el sistema de vigilancia . }\end{array}$ \\
\hline
\end{tabular}

Como citar este artículo: J.A. Calderón, G. Amarillo, L. Silva., y C. Donoso, “Biometría dactilar: una nueva alternativa de controlar efectivamente la asistencia a clases", Investigación e Innovación en Ingenierias, vol. 6, n. 1, pp. 27 - 39., 2018. DOl:https://10.17081/invinno.6.1.2773 


\begin{tabular}{|l|l|}
\hline \multicolumn{2}{|c|}{ Reconocimiento de voz } \\
\hline \multicolumn{1}{|c|}{ Ventajas } & \multicolumn{1}{c|}{ Desventajas } \\
\hline $\begin{array}{l}\text { Puede ser confiable es una marca } \\
\text { indibidual de una persona. }\end{array}$ & $\begin{array}{l}\text { La voz puede ser grabada después para } \\
\text { engañar al sistema }\end{array}$ \\
\hline \multirow{2}{*}{ Es un sistema biométrico aceptado. } & $\begin{array}{l}\text { Puede ser alterada por edad o por } \\
\text { enfermedades o acciones como } \\
\text { resfriados o en algunos casos el } \\
\text { elstado de animohace que varien. }\end{array}$ \\
\cline { 2 - 2 } & $\begin{array}{l}\text { Su baja distintividad y es muy fácil de } \\
\text { ser imitada. }\end{array}$ \\
\hline
\end{tabular}

Tabla 2.

Ventajas y desventajas de reconocimiento de voz.

Fuente: [7]

elaboración propia

\begin{tabular}{|l|l|}
\hline \multicolumn{1}{|c|}{ Ventajas } & \multicolumn{1}{c|}{ Desventajas } \\
\hline $\begin{array}{l}\text { El iris tiene caracteristicas únicasque no } \\
\text { cambian con el tiempo. }\end{array}$ & $\begin{array}{l}\text { Para obtener la medida exacta el patrón } \\
\text { debe estar ubicado a una distancia } \\
\text { considerable des escáner, lo cual significa } \\
\text { incomodidad para el usuario. }\end{array}$ \\
\hline $\begin{array}{l}\text { El reconocimiento del iris es una técnica } \\
\text { donde el usuario puederealizar la } \\
\text { verificación desde una distancia cómoda } \\
\text { del escáner. }\end{array}$ & $\begin{array}{l}\text { Este sistema es poco utilizado en el } \\
\text { mercado por lo que no es de fácil } \\
\text { adquisición, }\end{array}$ \\
\hline $\begin{array}{l}\text { El tiempo de verificación de retina es } \\
\text { rápido. }\end{array}$ & Necesidad del desarrollo del algoritmo \\
\hline \multirow{2}{*}{$\begin{array}{l}\text { El análisis de retina es dificil de engañar, } \\
\text { no existe la manera de hacer una réplica } \\
\text { igual y cuando la persona fallece la retina se } \\
\text { deteriora. }\end{array}$} & $\begin{array}{l}\text { El reconocimiento de retina puede } \\
\text { revelar enfermedades y afecciones las } \\
\text { cuales pueden vulnerar el derecho } \\
\text { ala intimidadde los usuarios como } \\
\text { hipertensión }\end{array}$ \\
\cline { 2 - 3 } & $\begin{array}{l}\text { El reconocimiento de retina la captura } \\
\text { de este rango biométrico es compleja ya } \\
\text { que se necesita voluntad y cooperación } \\
\text { por parte del usuario ya que se requiere } \\
\text { contacto con el sensor y su gran } \\
\text { aceptabilidad es muy importante. }\end{array}$ \\
\cline { 2 - 2 } & $\begin{array}{l}\text { El reconocimiento del iris tecnologia } \\
\text { muy costosa }\end{array}$ \\
\hline
\end{tabular}

Tabla 3.

Ventajas y desventajas de reconocimiento por iris Fuente: [7] Elaboración propia.

Con la comparación realizada, se propuso la implementación de tecnología biométrica según las necesidades planteadas por los estudiantes y profesores de la Universidad UNINPAHU a través de encuestas, observaciones, reuniones y charlas. Así se determinó la mejor solución y, lo más importante, la factibilidad del desarrollo y la posterior implementación de un software que automatiza este proceso.

En este proceso investigativo y de desarrollo tecnológico se ha usado un tipo de investigación exploratoria, mediante el que se concluyó que la biometría era la mejor solución [8].

Como citar este artículo: J.A. Calderón, G. Amarillo, L. Silva., y C. Donoso, "Biometría dactilar: una nueva alternativa de controlar efectivamente la asistencia a clases", Investigación e Innovación en Ingenierias, vol. 6, n. 1, pp. 27 - 39., 2018. DOl:https://10.17081/invinno.6.1.2773 


\section{Aplicación de instrumentos}

La población para este estudio y proceso de encuestas corresponde a los estudiantes y profesores de la Fundación Universitaria INPAHU, la cual estuvo constituida por cerca de 3500 estudiantes y profesores en la sede de Bogotá.

De toda ella se seleccionó una muestra de 55 estudiantes y docentes de la jornada nocturna, en tanto que la recolección de datos fue realizada entre los meses de septiembre y octubre de 2016.

El muestreo utilizado se basó en la media, ya que se pretende estimar el tamaño de la muestra [9], para cuyo cálculo exacto se utilizó la siguiente fórmula de la distribución gaussiana [10]:

\author{
Fórmula \\ Gaussiana \\ Fuente: [10].
}

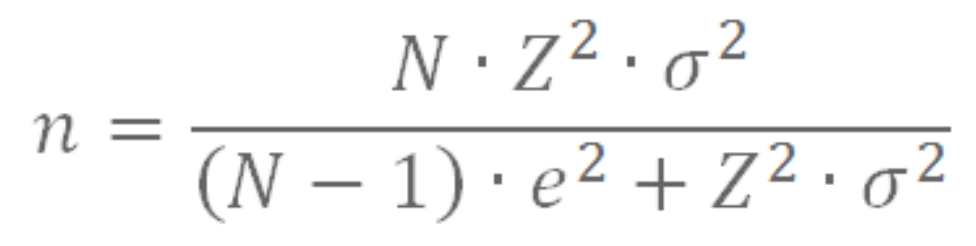

Donde: $\sigma 2$ es la varianza que se espera encontrar en la población (es decir, el cuadrado de la desviación estándar, $\sigma$ ). Este dato debe obtenerse de un estudio previo o de una estimación propia que para nuestro caso es de (10).

$\mathrm{N}=$ Tamaño del universo (3500)

$Z=$ Es la desviación del valor medio aceptado para lograr el nivel de confianza deseado. En función del nivel de confianza buscado, se usa un valor determinado que viene dado por la forma que tiene la distribución de Gauss. Los valores más frecuentes al respecto son:

Nivel de confianza $90 \%->Z=1,645$

Nivel de confianza $95 \%->Z=1,96$

Nivel de confianza $99 \%$-> Z=2,575

Para nuestro caso fue del $95 \%$

e = Es el margen de error máximo, que en esta investigación fue de (3).

n = El tamaño de la muestra a calcular: que podía ser de 42 personas pero aquí llegó a 55. Lo anterior tomando en cuenta que con 42 personas encuestadas, en un $95 \%$ de veces el dato real que se busca, tendrá un $1 \%$ de error con base en el resultado de las encuestas.

Como ya se dijo, la recolección de los datos se realizó a través de encuestas, elaboradas sobre la percepción del control de asistencia, que consta de 12 preguntas para medir el sistema de control de asistencia, el gasto en papelería y el uso, como medio alternativo, de un sistema biométrico para la toma de asistencia. 
Para representar el análisis de resultados se escogió como la gráfica de tarta, dada su facilidad de comprensión, y que son utilizadas para exponer cualquier tipo de información. Una gran ventaja de este tipo de gráficos es que la información resultante es visiblemente clara.

El análisis de cada pregunta evidenció que la percepción de los estudiantes acerca del control actual de asistencia a clase es el siguiente: regular, un $45 \%$; malo, 10\%. Esto indica que más del 50\% de la población encuestada percibe falencias en el sistema de asistencia actual.

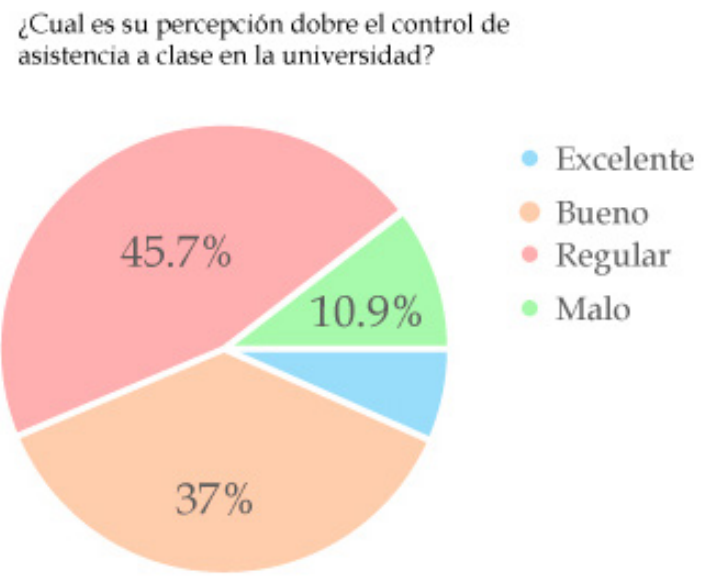

Figura 1.

Pregunta 1 de encuesta Fuente: Autores.

Por otra parte, cuando se indagó si la asistencia a clase se tenido que tomar más de una vez por pérdida de la información, se hallaron los siguientes resultados: $13 \%$ muchas veces, $23.9 \%$ pocas veces; y $32.6 \%$ alguna vez, confirmando que más del $50 \%$ de la muestra ha tenido que volver a diligenciar un formato de asistencia por perdida de la información al menos en una ocasión.

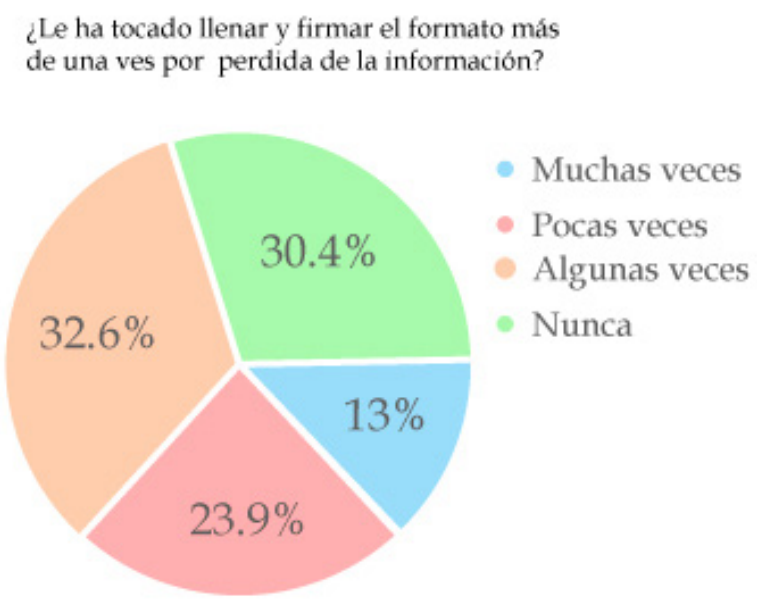

\section{Figura 2.}

Pregunta 2 de encuesta Fuente: Autores. 
En los casos en que el estudiante por algún inconveniente olvida llenar la asistencia se halló que por lo menos un $67 \%$ ha sufrido este inconveniente.

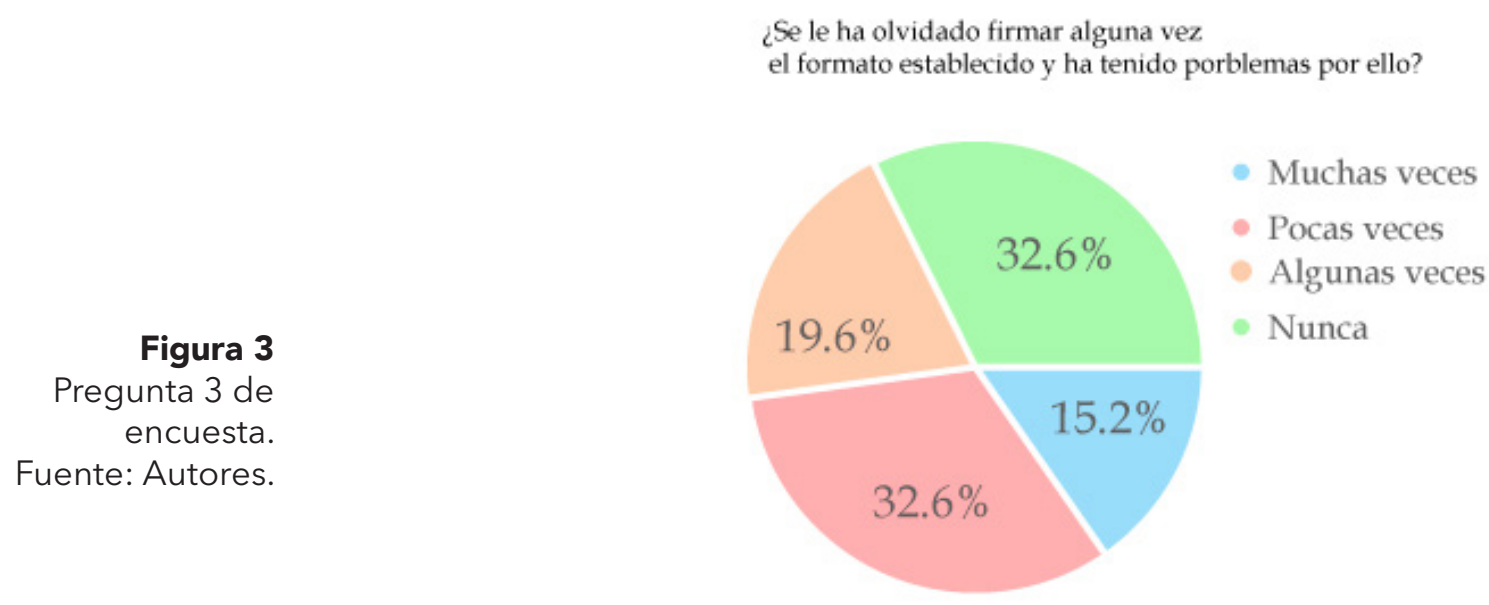

Respecto a la percepción en gasto de papelería, un $87 \%$ de estudiantes se encontraban de acuerdo con el gasto en papelería, siendo este un porcentaje considerable y el cual nos da un indicio que es un punto importante para la ejecución del proyecto.

Figura 4

Pregunta 4 de

encuesta

Fuente Autores.

¿Esta deacuerdo que se desperdicia mucho papel con los formatos de asistencia?

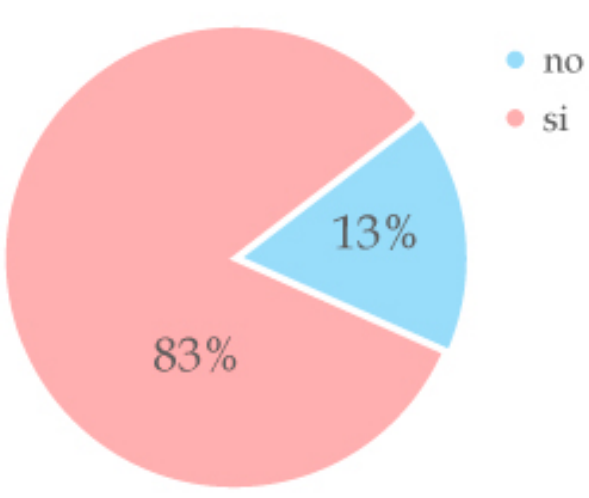

Con relación al uso de medios alternativos como la biometría para el control de asistencia a clase, se encontró que un 80.4 \% de estudiantes estaban de acuerdo, siendo esta pregunta la base que llevó a ejecutar el proyecto. 


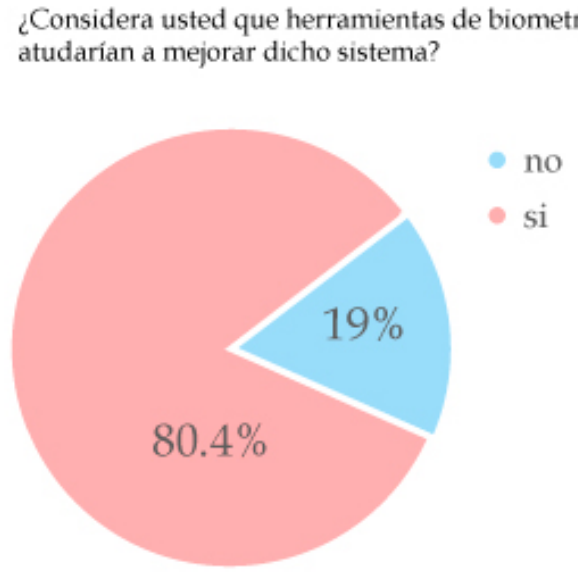

\author{
Figura 5. \\ Pregunta 5 de \\ encuestas \\ Fuente: Autores.
}

En términos generales, se llegó a la conclusión de que la manera como la Universidad lleva el control de la asistencia, a pesar de ser eficiente, no es la más recomendada por motivos expuestos anteriormente.

\title{
Metodología Scrum para el desarrollo del software
}

En la concreción del proyecto, aparte de usar una metodología de investigación, se tuvo que elegir una metodología de desarrollo de software ágil para el caso SCRUM, que facilita el proceso y ayuda con las mejores prácticas y seguimiento al control de actividades, eniendo en cuenta que una metodología ágil de desarrollo no significa que se produzca un software en corto tiempo, pero garantiza una metodología sin tantas interrupciones, enfocada en lo más importante del proyecto [11].

Una de las ventajas de usar metodologías ágiles es la facilidad del proceso de cambio a lo largo del ciclo de vida del proyecto y del desarrollo como tal, ya que se manejan procesos iterativos o llamados sprint, en los cuales cada iteración es como un mini proyecto, que contiene piezas de ese proyecto macro que es el proyecto completo. En estas iteraciones se maneja una importante recolecta de requisitos, que tienen la gran ventaja de ser susceptibles de modificación en caso necesario, utilizando las iteraciones y las necesidades que vayan surgiendo y evitando grandes pérdidas en cuanto a costos, tiempo y hasta en la motivación de los actores que influyen en el proyecto.

Otra ventajas es la visibilidad de los avances en tiempos menores a las metodologías normales, ya que las entregas realizadas en una iteración pueden durar entre 1 y 5 semanas máximo, lo que da pie para revisar si el proyecto va por buen camino y se puede intervenir a tiempo para realizar modificaciones sin mayor impacto para el proyecto y darle a este un mejor horizonte.

También se puede incluir entre estas ventajas la simplicidad del trabajo en equipo que nos brinda es-

Como citar este artículo: J.A. Calderón, G. Amarillo, L. Silva., y C. Donoso, "Biometría dactilar: una nueva alternativa de controlar efectivamente la asistencia a clases", Investigación e Innovación en Ingenierias, vol. 6, n. 1, pp. 27 - 39., 2018. DOI:https://10.17081/invinno.6.1.2773 
tas metodologías ágiles de desarrollo, que se dan al eliminar el trabajo innecesario y concentrarse en el trabajo realmente importante para conseguir resultados de buena calidad en tiempos menores. Un ejemplo de esto es que las metodologías ágiles no incluyen toda la documentación y procesos que se deben considerar en un proyecto con metodología clásica. En este sentido es claro que la documentación es importante, pero en las metodologías ágiles solo se toma la necesaria para el proyecto y se elimina mucho de la que puede convertirse en una piedra en el zapato para la continuación fluida de un proyecto. Es por esto que un proyecto de software realizado con metodologías tradicionales puede durar mucho tiempo en realizarse, y asimismo sus primeros resultados salen a la luz después de mucho tiempoy si se hallan necesidades que lo requieran cambios, se perdería mucho esfuerzo, trabajo, dedicación, y costos superiores.

Para el proceso práctico se identificaron y se reconocieron los roles, comenzando un proceso de estudio de cada rol para su desempeño. En este marco, con el fin de aplicar una metodología lo más próxima a Scrum, se procedió a realizar un plan de trabajo, el cual se resume a continuación.

Como primera fase se procedió al levantamiento de requerimientos por parte del Scrum Master por medio de historias de usuario que sirvieron para trazar la ruta a seguir.

Con el fin de plasmar la información en una herramienta tecnológica y realizar seguimiento a esta, se utilizó, además, el Visual Studio Team Foundation para llevar el control del proceso ágil, por tanto se subieron las historias de usuario a dicha plataforma. Con las historias de usuario ya en plataforma, se realizó una reunión para priorizar y dar puntos a cada historia de usuario con el fin de establecer cuáles serían tomadas en cuenta para el primer sprint. En este proceso se tomó en cuenta la complejidad de cada historia, el tiempo que podía tardar en realizarse y la fecha de finalización del primer sprint. Esta última se acordó para un mes después de realizado el sprint de iniciación [12 - 14].

Normalmente en la metodología Scrum, se llevan a cabo unos Dealy Meeting o sprint diarios que no deben superar 15 minutos, pero, debido al tiempo con el que se contó para la ejecución del proyecto no era posible seguir este principio, dado a esto se establecieron reuniones cada dos días, de 15 minutos sin tener en cuenta los fines de semana. Estas reuniones eran acordadas con el grupo y ayudaron a ver el avance del proyecto y a hacer seguimiento a las dificultades que se estaban presentando como grupo en el desarrollo del proyecto.

Una vez culminado el primer sprint se procedió a realizar una reunión de finalización del sprint y se realizó una entrega funcional de las primeras historias de usuario. Este mismo día se también se realizó una reunión de retrospectiva y se tomaron los puntos más problemáticos con el fin de mejorarlos para el siguiente sprint.

Luego se realizó de nuevo una reunión de iniciación para el segundo y último sprint con el fin de incluir las historias de usuario faltantes. Para este segundo sprint se estableció nuevamente fecha de finalización y objetivo a realizar, así como la continuidad de las reuniones cada dos días hasta la finalización de este. 


\section{Resultados}

Todo el conjunto de herramientas tecnológicas lleva sin duda a mejorar la actividad y el proceso de control de asistencia de alumnos y profesores en las aulas de clase de la Universidad UNINPAHU, evitando los problemas de riesgo de veracidad de la información suministrada por medio de papel. El funcionamiento que se planteó para resolver la problemática en el control de asistencia es el uso de la biometría y un software que en grandes rasgos funciona por medio de un sistema. Mediante este se ejecuta la captura de la huella del estudiante como forma de registro, con la que se almacenan los patrones o características a analizar y una imagen a valorar para gestionar un registro; luego se realiza un proceso de comparación de las características extraídas por el sistema y se convierten en un identificador numérico.

Esta salida da como resultado una similitud para tomar una decisión que en caso de ser positiva procede a permite el acceso y marcaje de asistencias. Todo esto apoyado en una plataforma que gestiona ingresos, consultas de cursos, consulta de grupos, registros y reportes, que en su totalidad dan forma al sistema de control de asistencia.

\section{Discusión}

Después del proceso de investigación surge la siguiente pregunta: ¿Podrá la aplicación, unida con la tecnología de biométricos, ayudar y mejorar el proceso de control de asistencia a clases de la Fundación Universitaria UNINPAHU? Al respecto, hay muchos factores que inducen hacia una respuesta positiva, pero todo depende de la forma en que las personas asimilen el cambio del proceso, así como de la capacidad de la universidad para implementar esta solución.

Por otra parte, la implementación es claramente viable pero también depende de los requerimientos del sistema y de una infraestructura adecuada para disponer de dispositivos necesarios en la lectura de la huella dactilar en cada salón. Es importante precisar en este sentido que esta variable puede ser mejorada de acuerdo con los dispositivos concretos que se adquieran. 


\section{Conclusión}

Después de analizar los resultados de la problemática, se estableció que los estudiantes y profesores quieren cambiar el proceso de control a clase de la Fundación Universitaria UNINPAHU. En esta perspectiva:

- Se identificó que la biometría dactilar brinda seguridad y veracidad de la información.

- Se diseñó y se desarrolló, por medio de metodologías agiles, la aplicación para control de la asistencia a clase con ayuda de biométrico, en la Fundación Universitaria UNINPAHU.

- Se evidenció, por último, la disminución de tiempos en registro, disminución del uso de papel, centralización de los datos e información en línea. 


\section{Referencias}

1. M. Dominhez Aquino, C. Herrera y O. Jara, Programación de software biométrico (tesis de pregrado). Universidad de Guayaquil, Guayaquil, 2007.

2. J. E. G. Ricardo, Estudio de factibilidad para el control de acceso biométrico (tesis de pregrado). Universidad de La Salle, Bogotá, 2007.

3. A. M. Bolívar Cortés y A. Correa Monteiro, Creación de una unidad de negocio orientada hacia la implementación de los productos y servicios biométricos en las residencias de Bogotá, Colombia. Bogotá, 2009.

4. J. d. G. d. M. -. P. d. 1. N. Thill Eduardo, Biometría 2. Buenos Aires, 2011.

5. $\quad$ F. Serratosa. La biometría para la identificación de las personas. Cataluña, 2016.

6. W. Herschel, «The Origin of Finger-Printing, Oxford University Press,» 1916. [En línea]. Available: http://galton.org/fingerprints/books/herschel/herschel-1916-origins-1up.pdf.

7. Sanchez, C. El Espectador, «Registraduria Nacional del Estado Civil - Biometría», 2011. [En línea]. Available: http://www.registraduria.gov.

8. D. S. B. Rivero, Metodologías de la investigación. Shalom, 2008.

9. C. Ochoa, «Que tamaño de muestra necesito? - NetQuest,» 2013. [En línea]. Available: http://www. netquest.com/blog/es/que-tamano-de-muestra-necesito.

10. A. Guerrero Peña, La distribución normal o de Gauss en estadística básica. Bogotá, 2007.

11. SCRUMstudy, Una guía completa para el cuerpo de conocimiento de scrum, Phoenix, Arizona: SCRUMstudy, 2016.

12. msdn.microsoft, «msdn.microsoft,» junio 2016. [En línea]. Available: https://msdn.microsoft.com/esco/library/z1zx9t92.aspx.

13. M. Parra and S. Dúran, "Desarrollo organizacional y estrategias de tecnoformación en instituciones de educación superior colombianas", Dictamen Libre, no. 1415, pp. 14-23, 2014.

14. E. Orozco Acosta, «Simulación en tiempo discreto de un proceso de abastecimiento de combustible como una herramienta de toma de decisiones: Caso estación de servicios en Barranquilla,» Dictamen Libre, nº 10/11, pp. 14-23, 2012. 